\title{
Quadrature Detection of Ultrasound-Modulated Photons with a Gain-Modulated, Image-Intensified, CCD Camera
}

\author{
David J. Hall*, Ulas Sunar and Salman Farshchi-Heydari
}

Department of Radiology, University of California, San Diego, CA 92093-0819, USA

\begin{abstract}
Acousto-optic imaging promises to provide in vivo images of optical contrast but with the superior spatial resolution of ultrasound imaging. Here we present novel quadrature detection of ultrasound-modulated photons with a gainmodulated, image-intensified, CCD camera. The additional detection of ultrasound-modulated fluorescence photons demonstrates potential for in vivo acousto-optic molecular imaging.
\end{abstract}

Keywords: Acousto-optic, fluorescence, ultrasound.

\section{INTRODUCTION}

In the last several years there has been rapid growth in the optical molecular imaging of small animals in vivo [1]. Longitudinal studies can now be performed employing optical probes based on bioluminescence and fluorescence markers [2]. Nevertheless, optical imaging suffers from the inherent high scattering of optical photons by biological tissue resulting in centimeter spatial resolution for applications such as optical breast imaging [3]. To address this issue researchers are combining optics and ultrasound to provide optical images at superior ultrasound spatial resolution $(\mathrm{mm})$. One popular approach is opto-acoustic imaging [4] which provides optical absorption images at ultrasound resolution. An alternative combination of optics and ultrasound is acousto-optic imaging [5]. This approach involves the modulation of photons, at the ultrasound frequency, which propagate through a small ultrasound focal zone. Optical images generated from only these ultrasound-modulated photons will thus have the improved spatial resolution of the ultrasound focal zone. The main challenge is the detection and discrimination of ultrasound-modulated photons from the overwhelming presence of non-modulated photons not passing through the ultrasound focal zone. To date, most researchers have employed coherent light sources and detecting ultrasound-modulated speckles [6,7]. To increase the SNR of the modulated signal a large number of speckles must be detected. However, since the detected speckles modulate at the same frequency but with random phase, a direct spatial integration of the speckles would lead to a loss of modulation. Instead, parallel detection techniques employ a coherent light source modulated at the ultrasound frequency and detect the ultrasound-modulated speckles with a CCD camera [8]. Quadrature detection, i.e. acquiring CCD images of speckles at four different phase shifts between the laser and ultrasound modulation, permits the modulation amplitude of each speckle to be determined which summed

*Address correspondence to this author at the Department of Radiology, University of California, San Diego, CA 92093-0819, USA;

E-mail: djhall@ucsd.edu over all speckles leads to an increase in SNR. Researchers have also elucidated on the physical process of ultrasoundmodulation of coherent photons $[9,10]$. The requirement for coherence limits the choice of light sources to those with a coherence length at least as long as the optical pathlength (several centimeters for highly scattering biological tissue), as well as high imaging spatial resolution (order of microns) to resolve individual speckles, and prohibits the modulation of incoherent processes such as fluorescence.

More recently researchers have reported detection of ultrasound-modulated incoherent photons [11]. Here, the ultrasound modulates the optical properties (refractive index, absorption, scattering) of the ultrasound focal zone resulting in a modulation of the local optical attenuation coefficient causing an in-phase intensity modulation of the photons (be they incoherent or coherent). In essence, it can be considered as generating a modulated light source at the ultrasound frequency in the focal zone. The detection of the in-phase intensity modulation does not require the high imaging spatial resolution needed to resolve the random phase speckles from coherent photons and permits direct spatial integration of the detected photons. Here we present our novel parallel detection approach and preliminary results demonstrating detection of ultrasound-modulated photons and fluorescence.

\section{METHOD}

The detection of ultrasound-modulated photons has employed photomultiplier tubes (PMTs) which have adequate bandwidth to measure the $\mathrm{MHz}$ modulation frequency but limited detection area. Unfortunately larger area detectors, such as CCD cameras, have inadequate frame rates $(\mathrm{Hz})$ to capture the $\mathrm{MHz}$ signal. However, CCD cameras have been employed in parallel detection quadrature methods by crosscorrelating the ultrasound modulation with a laser modulated at the ultrasound frequency and acquiring CCD images at four different phase shifts [8]. We have developed an alternative, novel, parallel quadrature detection approach where the laser is steady-state and the ultrasound modulation is cross-correlated with an image-intensified CCD camera which is gain-modulated at the ultrasound frequency and CCD images are acquired at four different phase shifts. In 


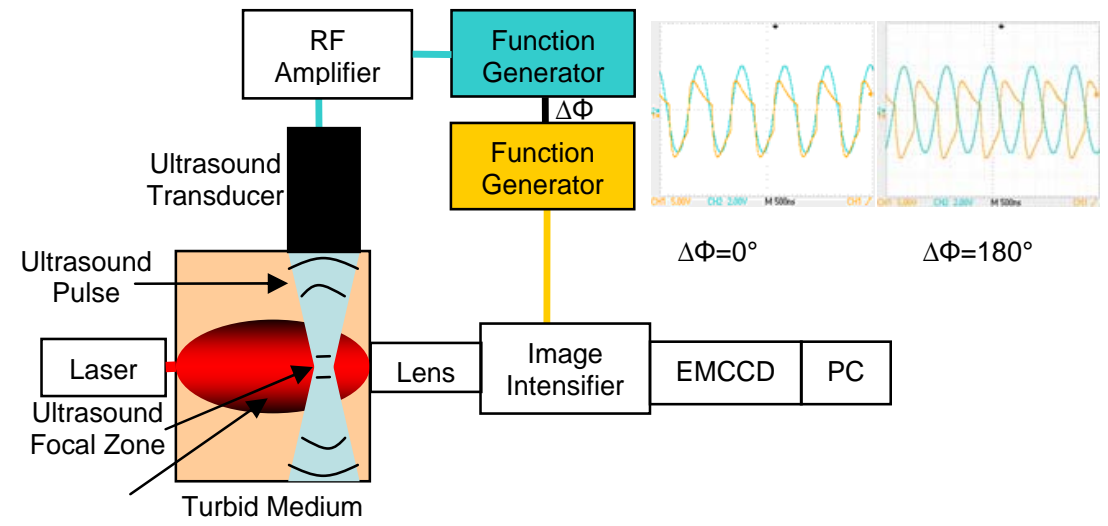

Fig. (1). Configuration for detection of ultrasound-modulated photons with a gain-modulated, image-intensified, CCD camera. $1 \mathrm{MHz}$ driving signals for US transducer and Image Intensifier gain shown (top right) for relative phase shifts of $0^{\circ}$ and $180^{\circ}$.

other words, we are cross-correlating the ultrasound and the optical detector using a steady-state source as opposed to cross-correlating the ultrasound and the optical source using a steady-state detector [8]. The experimental configuration is shown in Fig. (1).

In this setup we employed a laser diode $(5 \mathrm{~mW}$ at 650 $\mathrm{nm})$ to illuminate a water tank (65 mm wide) to which we added varying amounts of intralipid as an optical scatterer. Light was detected with a gain-modulated image intensifier (Picostar HRI, LaVision, Germany) with a highly sensitive electron-multiplying CCD camera (Andor, CA) to detect the output of the image intensifier and store it on the acquisition computer. Modulation of the image intensifier gain was achieved with a function generator (Stanford Research Systems, DS345) outputting a sine wave at the ultrasound frequency and phase-locked to a second identical function generator input to an RF Amplifier (ENI, 240L) to drive a single-element ultrasound transducer (Panametrics, V303) with a $1 \mathrm{MHz}$ sine wave $(50 \mathrm{Vpp})$ with 20 pulses $(20 \mu \mathrm{s}$ pulselength) every millisecond ( $2 \%$ duty cycle). The phase shift, $\Delta \Phi$, between the image intensifier gain and ultrasound transducer can be varied from 0 to $2 \pi$. Fig. (1) shows the driving signals for the image intensifier gain (yellow) and the ultrasound transducer (blue) for the two conditions of $\Delta \Phi=0^{\circ}$ and $\Delta \Phi=180^{\circ}$. The ultrasound transducer was submerged in the water tank such that its focal zone (100 KPa peak pressure) intercepted the line-of-sight between the laser diode $(\sim 0.1$ $\mathrm{mW}$ in focal zone) and the image intensifier in transmission mode. A $50 \mathrm{~mm}$ f/1.4 Lens (Nikon) permitted photon detection over a large area (several $\left.\mathrm{cm}^{2}\right)$.

\section{RESULTS}

\section{Quadrature Detection of Ultrasound-Modulated Photons}

The image intensifier's microchannel plate (MCP) was set to a voltage of $260 \mathrm{~V}$ (low gain) and the CCD integration time was set to $320 \mathrm{~ms}$ per frame. Several images were then acquired for $\Delta \Phi=0^{\circ}$ before switching to $\Delta \Phi=180^{\circ}$ and finally with the ultrasound turned-off as a control. For quadrature detection we generally require four phase measurements. However, here we intentionally phase-aligned the signals for the special case that the maximum and minimum signals coincided with $\Delta \Phi=0^{\circ}$ and $\Delta \Phi=180^{\circ}$ respectively. As such, the amplitude of the acousto-optic effect is simply the differ- ence between the two measurements. Each CCD image was spatially integrated, since the intensity modulation was inphase, and the intensity values, I, were plotted (Fig. 2). Note, similar to other researchers [8], this approach would also be applicable to parallel quadrature detection of ultrasoundmodulated speckles from coherent photons.

Fig. (2) shows that ultrasound-modulation causes a decrease in the local optical attenuation when $\Delta \Phi=0^{\circ}$ resulting in higher intensity, and an increase in the local optical attenuation when $\Delta \Phi=180^{\circ}$ resulting in lower intensity, compared to the local optical attenuation and intensity when no ultrasound is applied. We continued to switch the detector's phase back and forth when no ultrasound was applied as a further control. Also it was noted that the intensity obtained without ultrasound being applied is midway between $\Delta \Phi=0^{\circ}$ and $\Delta \Phi=180^{\circ}$ as expected. The modulation in the optical intensity due to ultrasound can best be represented by the induced modulation depth, $\mathrm{M}$, equal to $\left(\mathrm{I} \Delta \Phi 0^{\circ}\right.$ $\left.\mathrm{I} \Delta \Phi 180^{\circ}\right) /\left(\mathrm{I} \Delta \Phi 0^{\circ}+\mathrm{I} \Delta \Phi 180^{\circ}\right)$ which here is approximately $3 \%$.

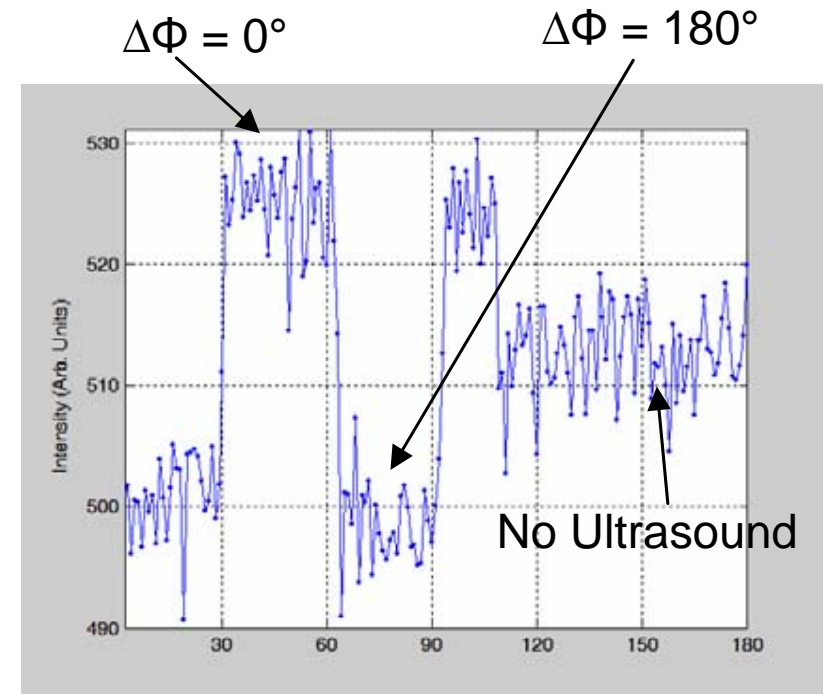

Fig. (2). Detection of ultrasound-modulated photons with $3 \%$ modulation depth. Recorded Intensity for relative phase shifts of $0^{\circ}$ and $180^{\circ}$ with and without ultrasound applied. 


\section{Quadrature Detection of Ultrasound-Modulated Fluores- cence}

To investigate whether we could detect ultrasoundmodulated fluorescence we used a small cylindrical fluorescent pellet, $15 \mathrm{~mm}$ diameter by $8 \mathrm{~mm}$ height, containing the fluorophore Qdot800 (Invitrogen, CA) at $10 \mathrm{pM}$ concentration. Our manufacture of such pellets is reported elsewhere [12]. The fluorescent pellet was placed on the side of the water tank in the line of sight between the laser diode and optical detector to which we had added a wavelength filter (OmegaFilters, 780 LP) to block laser excitation light and permit detection of fluorescence from the fluorescent pellet. Due to the weak fluorescence signal we increased the MCP voltage to $510 \mathrm{~V}$ (medium gain) and acquired several images with an integration time of $320 \mathrm{~ms}$ at $\Delta \Phi=0^{\circ}$ before switching to $\Delta \Phi=180^{\circ}$. The resultant CCD images were then integrated and the intensity values are displayed in Fig. (3).

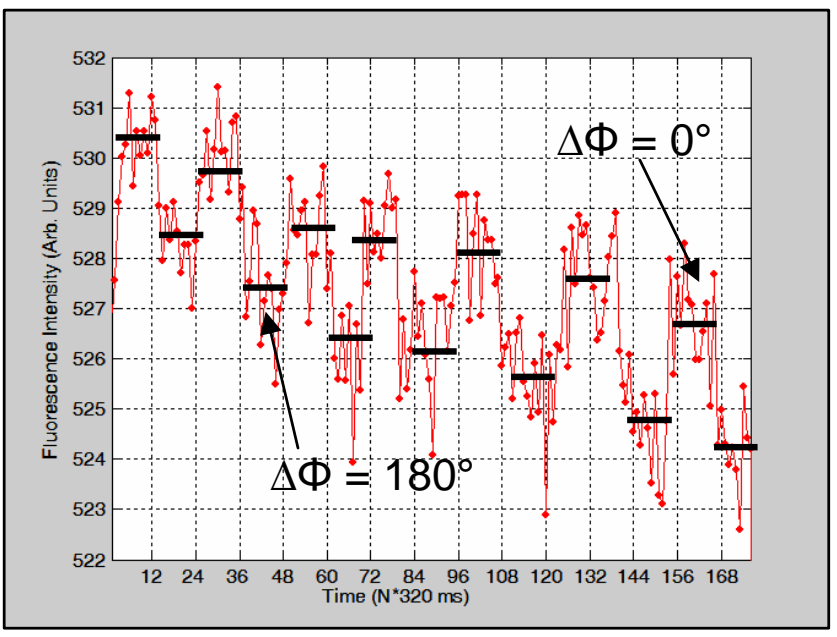

Fig. (3). Detection of ultrasound-modulated fluorescence. Recorded Fluorescence Intensity for relative phase shifts of $0^{\circ}$ (peaks) and $180^{\circ}$ (troughs).

This result demonstrates the detection of ultrasoundmodulated fluorescence with the gain-modulated image intensifier. Note the modulation depth is much weaker, approximately $0.2 \%$, compared to that measured from excitation photons (Fig. 2). Systematic downwards drift is due primarily to small laser and detector drifts we observed during our measurements with and without ultrasound modulation. This drift was not a concern since modulation depth was the main interest and simple data-processing can remove this baseline effect. As before, without the ultrasound being applied there was no modulation detected. It should be noted that the fluorescent pellet is actually located just after the ultrasound focal zone and closer to the detector to ensure a strong detected optical signal, rather than submerged within the focal zone itself. As such, the fluorescence photons are not being modulated per se, but rather the excitation photons from the laser diode are being modulated just before the pellet which then induces a modulation in the fluorescence signal. Indeed, even if the fluorescent pellet and focal zone were exactly co-localized the fluorescence modulation would be due to modulation of both the excitation photons and emitted fluorescence photons. Again it is useful to consider the continuous wave photons from the optical source as forming a localized modulated optical source in the ultra- sound focal zone which, when in close proximity of a fluorophore, generates a modulated fluorescence signal which would otherwise be a steady-state fluorescence signal. Hence, there is potential to image fluorophore distribution with ultrasound spatial resolution. It should be noted that a slight misalignment in the ultrasound focal zone proximity to the fluorescent pellet led to a total loss of the modulated fluorescence signal demonstrating that the method should provide a very high spatial resolution $(\sim \mathrm{mm})$.

Beyond the quadrature detection of ultrasound-modulated photons demonstrated here, we plan to perform acousto-optic imaging with this detection approach. The laser and ultrasound focal zone will be raster-scanned across an optically scattering object and the amplitude of the ultrasoundmodulated photons measured for each scan position to provide an image. For a homogeneous object we expect constant amplitude, whereas scanning across a more optically attenuating inhomogeneity we expect a decrease in amplitude. Alternatively, in fluorescence imaging mode scanning across a fluorescent inhomogeneity we expect an increase in amplitude due to the detection of ultrasound-modulated fluorescence photons. Since only photons within the small ultrasound focal zone are modulated and contribute to the acousto-optic images we expect much higher image spatial resolution than achievable from optical imaging which employs all detected photons.

\section{CONCLUSIONS}

This work has presented the novel quadrature detection of ultrasound-modulated photons with a gain-modulated, image-intensified CCD camera. Furthermore, the detection with this novel approach of ultrasound-modulated fluorescence, an incoherent process, offers the opportunity for acousto-optic molecular imaging employing fluorescencebased probes. Future work will involve a scanning configuration to perform acousto-optic imaging.

\section{ACKNOWLEDGEMENTS}

The authors gratefully acknowledge a DOD Army PCRP Idea Development award (W81XWH-07-1-0125) for funding this work, support from GE Healthcare, and useful discussions with Dr. Robert Mattrey (UCSD) regarding ultrasound.

\section{REFERENCES}

[1] Weissleder R, Ntziachristos V. Shedding light onto live molecular targets. Nat Med 2003; 9: 123-8.

[2] Rice B, Cable M, Nelson M. In Vivo Imaging of Light-emitting Probes. J Biomed Opt 2001; 6(4): 432-40.

[3] Intes X, Djeziri S, Ichalalene Z, et al. Time-domain optical mammography SoftScan: Initial results. Acad Radiol 2005; 12(8): 93447.

[4] Oraevsky A. Detection, diagnostics and image-guided therapy of cancer using laser optoacoustic imaging system and gold nanoparticles. IEEE 2004; 250-51.

[5] Wang L. Ultrasound-mediated biophotonic imaging: A review of acousto-optical tomography and photo-acoustic tomography. Dis Markers 2003; 19: 123-38.

[6] Wang L, Jacques S, Zhao X. Continuous-wave ultrasonic modulation of scattered laser light to image objects in turbid media. Opt Lett 1995; 20(6): 629-31.

[7] Li J, Ku G, Wang L. Ultrasound-modulated optical tomography of biological tissue by use of contrast of laser speckles. Appl Opt 2002; 41(28): 6030-5. 
[8] Leveque S, Boccara A, Lebec M, Saint-Jalmes H. Ultrasonic tagging of photon paths in scattering media: parallel speckle modulation processing. Opt Lett 1999; 24(3): 181-3.

[9] Wang L. Mechanisms of Ultrasonic modulation of multiply scattered coherent light: An analytical model. Phys Rev Lett 2001; 87(4): 043903.

[10] Zemp R, Sakadzic S, Wang L. Stochastic explanation of speckle contrast detection in ultrasound-modulated optical tomography. Phys Rev E 2006; 73: 061920.
[11] Krishnan K, Formitchov P, Lomnes S, Kollegal M, Jansen F. A theory for the ultrasonic modulation of incoherent light in turbid medium. SPIE 2005; 6009: 147-58.

[12] Hall D, Han S. Preliminary Results from a multi-wavelength time domain optical molecular imaging system. SPIE 2007; 6430: 64300T.

(C) Hall et al.; Licensee Bentham Open.

This is an open access article licensed under the terms of the Creative Commons Attribution Non-Commercial License (http://creativecommons.org/licenses/by$\mathrm{nc} / 3.0 /$ ) which permits unrestricted, non-commercial use, distribution and reproduction in any medium, provided the work is properly cited. 